\title{
Optimal Allocation of Distributed Generation and Capacitor Banks using Probabilistic Generation Models with Correlations
}

\author{
Luan D. L. Pereira ${ }^{\mathrm{a}}$, Imene Yahyaoui ${ }^{\mathrm{b}}$, Rodrigo Fiorottic ${ }^{\mathrm{c}}$, Luíza S. de \\ Menezes $^{\mathrm{d}}$, Jussara F. Fardin ${ }^{\mathrm{d}}$, Helder R. O. Rocha ${ }^{\mathrm{d}}$, Fernando Tadeo \\ ${ }^{a}$ Federal Institute of Norte de Minas Gerais, Brazil \\ ${ }^{b}$ Higher School of Experimental Sciences and Technology, University of Rey Juan Carlos, \\ Madrid, Spain \\ ${ }^{c}$ Federal Institute of Espírito Santo, Brazil \\ ${ }^{d}$ Department of Electrical Engineering, Federal University of Espirito Santo, Brazil \\ ${ }^{e}$ Institute of Sustainable Processes, University of Valladolid, Spain
}

\begin{abstract}
The allocation of distributed generation and capacitor banks is critical for success in the planning of power grids. A methodology is developed for the optimal placement of distributed renewable generation (wind and photovoltaic powers) and capacitor banks is developed based on technical and economic parameters. In order to preserve the horoseasonal and stochastic dependence nature of the wind and solar power, the methodology uses a model that integrates the sequential Monte Carlo method and the diagonal band Copula model, integrating historical data of wind speed, solar radiation and feeder load from the region of study. An efficient algorithm based on Genetic Algorithms is proposed to implement the optimization. The algorithm validation demonstrates a reduction of up to $71.7 \%$ in annual losses of active power in the Bandeira feeder and $73.4 \%$ in the Recife feeder, with adequate voltage levels and a return on investment of 6-7 years."
\end{abstract}

Keywords: Generation forecast, stochastic correlation, distributed generation, capacitor banks, genetic algorithm.

\section{Introduction}

The renewable energies that are currently most installed are photovoltaic and wind power, whose primary sources of energy are intermittent and 
stochastic [1]. In terms of Electric Power Systems (EPS), they are considered Distributed Generation Units (DG), defined as electric power sources connected directly to the distribution network or at the consumer measurement site [2]. They are implemented as presented in Figure 1. This introduction of DG into the electric distribution system generates new issues related to the energy quality and operating costs in the technical planning of EPS.

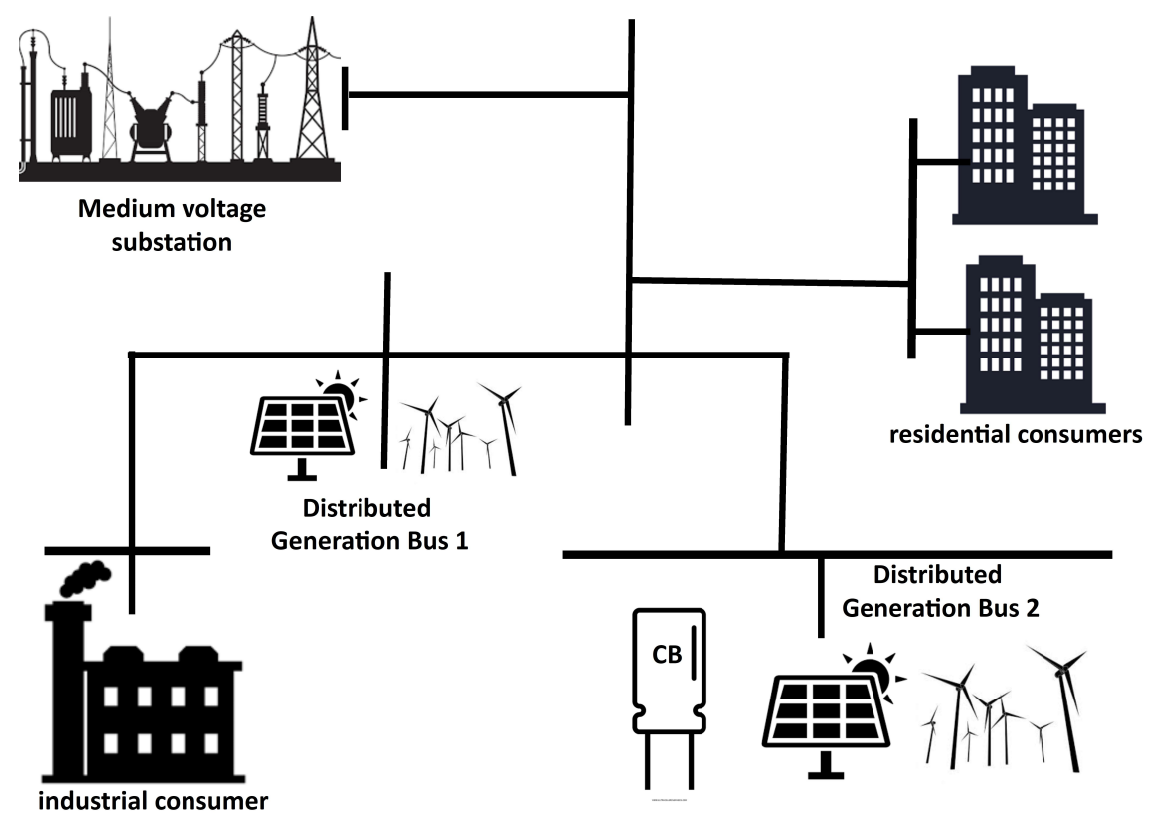

Figure 1: Architecture of the Electric Power Systems considered.

Medium and long-term load forecasting are important tools for the planning of the distribution network 3. These tools provide information necessary for the network and distribution line planning, as well as the work required for its reinforcement and extension [4]. However, the connection of DG makes the prediction of the demanded power a difficult task along the feeders [5], which in turn makes it necessary to apply an approach that incorporates the non-deterministic nature of the power generated by the DG. The incorporation of prediction uncertainties into the system management is necessary for the optimal operation of systems with a high penetration of these energy sources [6]. Such uncertainties should take into account the correlation of such primary energy sources as wind speed for wind power and solar radiance for photovoltaic generation. 
In addition to the DG power generation forecast, the correct allocation of these generators in the electrical system is decisive in the planning of the EPS [7]. The allocation of DG, as well as their type and size, can significantly affect the technical, economic and environmental performance of distribution systems [8]. Thus, for the allocation of these units, voltage levels, economic viability, loss analysis, electric power quality, reliability and safety should all be considered. However, the insertion of generation in the feeder reduces the active power value, while the reactive power remains practically the same, i.e., it causes the system power factor to decrease. Systems operating with a low power factor result in high active power losses and poor voltage profile. So it is essential to perform the simultaneous optimal allocation of CBs and DG to improve system performance.

Therefore, the problem of selecting ideal locations for the installation of DG and CBs has multiple objectives and constraints. According to [7], with the proper allocation, it is possible to improve the system's reliability and energy quality, minimize investment and operating costs, and mitigate the harmful effects that power generation might create in the power quality. This problem of allocation combines different aspects. So, one attractive solution is to use meta-heuristic algorithms, which use randomness in the search for optimal solutions.

Meta-heuristic methods are generally stochastic approaches that use randomness in the search for optimal solutions and are applied to problems in which there is no previous information. In meta-heuristics, when a good solution is found, a new search is carried out, incorporating new information in order to find a new optimal solution. Although there are other methods that can be applied to mono and multi-objective problems, the meta-heuristic one best meets the characteristics of the problem cue to its ability to change and adapt. Thanks to this, meta-heuristic algorithms can optimize a large number of discrete, continuous parameters or combinations of them and perform simultaneous searches in various regions of the search space.

\subsection{Related Works}

Several works in the literature have addressed some of the problems that

have emerged. For instance, 9] reviewed types of DGs and [10] investigated the challenges arising from the presence of DGs in electrical grids and optimization methods applied to solve the stated allocation problem. Zakaria et al. [11] comprehensively reviewed the generic steps of stochastic optimizations in renewable energy applications, from the modeling of the uncertainties and 
sampling of relevant information. In [12], several conventional and metaheuristic methodologies to address the optimal DG planning problem are reviewed and compared.

In [13, the authors used the Quasi Oppositional Chaotic Symbiotic Organisms Search technique, which is a meta-heuristic method that optimizes the allocation of distributed generation units in radial distribution networks. The objective of this study is to optimally reduce the real power loss, improve the voltage profile, and increase the voltage stability, with demonstration on a grid with 33, 69, and 118-bus. In [14] the authors developed a decision-making procedure for efficient placement and sizing of energy storage system in a virtual power plant composed of wind turbine, photovoltaic system, curtailable loads and diesel generators. The objective was to minimize the overall cost of the virtual power plant within a fixed time horizon planning and to optimize the power and the energy of the plant. In [15], the authors studied the impact of the input data resolution on the optimal allocation and power management of controllable and non-controllable renewable energy sources distributed generation system. The optimization problem in that paper considers the optimal allocation of all distributed generations and the optimal power control of controllable distributed generations. The authors proposed the use of an artificial neural network to estimate the optimal output of controllable distributed generations, which significantly decreases the dimensionality of the optimization problem.

In [16] a multi-objective DG and CBs allocation was addressed, integrated with a distribution network reconfiguration using the improved particle swarm optimization method to reduce annual energy loss, peak power losses, and substation capacity, while also maintaining better feeder current and voltage profiles. On the other hand, 17 proposed an improved gray wolf algorithm to allocate DG, CB and voltage regulators to minimize the investment costs of coordinated equipment and maximize the benefits arising from the reduction of energy losses and the purchase of energy from the network.

A modified version of a teaching-learning optimization algorithm was proposed in [18] to reduce the power losses and energy interruption for the costumers. It considered simultaneous DG scheduling, reconfiguration of the distribution networks and capacitor sizing. [19] presented a model for the simultaneous allocation and sizing of CBs (fixed and switched) and DG (dispatchable and stochastic) to reduce investments and operating costs. To solve the proposed model, an algorithm combining Tabu Search and ChuBeasley Genetic Algorithms was proposed and evaluated. A multi-objective 
particle swarm optimization algorithm (MPSO) was used in [20] for the DG and $\mathrm{CB}$ allocation problem. The objective function encompassed technical, economic and environmental aspects. Moreover, an MPSO method was applied to the allocation problem in [21], showing that power losses can be reduced, while the voltage stability and profile can be improved. In [22], a heuristic algorithm based on the Harmony search algorithm (HSA) and the particle artificial bee colony algorithm was proposed to deal simultaneously with feeder reconfiguration, and the allocation and sizing of DG and CBs. [23. proposed Bi-level programming, in which the high level optimization defined the location and sizing of DG and CB, while soft open points were optimized with respect to costs. A lower level optimization then performs a 24-hour dispatching to minimize power losses. A water cycle algorithm was applied in [24] for the DG allocation problem, taking into account technical, economic, and environmental aspects. In [25], a simplified heuristic method was studied for this problem, aiming for simplicity in the implementation and application to large distribution systems. An analytical optimization method is developed in [26] to minimize interconnection costs. The optimization considered geographical data of the network topology for the generation landuse and network infrastructure, which are neglected in most works.

In [27], a multi-objective DG and CB allocation was addressed, integrated with distribution network reconfiguration, using the improved particle swarm optimization method to reduce annual energy loss, peak power losses and substation capacity, while also maintaining better feeder current and voltage profiles. On the other hand, [28] proposed an improved gray wolf algorithm to allocate DG, CBs and voltage regulators to minimize the investment costs of coordinated equipment, while maximizing the benefits arising from the reduction of energy losses and the purchase of energy. [29] used a hybrid optimizer for optimal placement and sizing of GDs and CBs. The proposed hybrid method has a high convergence speed and is not stuck in an optimal location. Two IEEE standard distribution systems were used to demonstrate the effectiveness of the methodology. There was an improvement of the results in the different conflicting objectives. In [30, allocation of CBs was presented for a microgrid with DG and different load levels. The objective function included minimizing the cost of energy losses, peak power losses and the cost of the capacitors. The results demonstrated the ability of the algorithm to reduce the losses and improve the voltage profile during injection and non-injection of reactive power. 


\subsection{Our Contributions}

This paper proposes a methodology for the simultaneous, optimal allocation of CBs and DG based on wind and photovoltaic energy in distribution feeders. Previous works in the literature have not performed this simultaneous allocation considering the effects of the stochastic correlation of historical data series. Moreover, predictions did not use data from different regions. Therefore, the main contributions of this work are: the integration of the Sequential Monte Carlo method and the diagonal band Copula to preserve the horoseasonal and stochastic dependence nature of the generation, as well as the use of an efficient algorithm based on a Genetic Algorithm. The effectiveness is evaluated in two real feeders.

\subsection{Paper Organization}

The rest of the article is organized in six sections: Section 2 presents the DG stochastic generation model, considering the stochastic correlation between solar irradiation, ambient temperature, wind speed and the system load. The algorithm for performing the radial load flow is described in Section 3. Section 4 contains the mathematical formulation of the optimization problem. Some simulation results are commented in Section 5, and conclusions are provided in Section 6.

\section{DG stochastic generation model considering the stochastic cor- relation}

To preserve the stochastic nature of solar irradiation and wind speed, probabilistic methods are used, including the Monte Carlo simulation, that have already demonstrated significant advantages [31]. The allocation of DG goes beyond the availability of primary energy sources, since their location critically affects distribution systems by increasing or decreasing the active power losses of the system [32]. Therefore, it is necessary to develop an optimization model that indicates the ideal place of penetration of the DG and CB units, for the planning of the EPS [33]. The main steps to be carried out in the proposed methodology are:

Algorithm 1: Proposed Methodology.

- Step 1) Obtain and treat the historical series of wind speed, solar irradiation and temperature in the region of study; 
- Step 2) Generate the cumulative distribution functions (CDF) from the mean and standard deviation of each historical series;

- Step 3) Obtain the correlation between wind speed and solar irradiation from the method of Diagonal Band Copulation;

- Step 4) Apply the Monte Carlo Simulation to obtain the probabilistic model of energy generation from DG;

- Step 5) Determine the power of the DG;

- Step 6) Determine the optimal location of the DG and CB units using a Meta-heuristic.

\subsection{Wind Speed and Solar Irradiation Modeling}

In order to carry out a technical analysis implementation of wind generators and photovoltaic panels, it is necessary to have information of the wind speed and solar irradiation characteristics in the studied region. In fact, wind speed modeling can be performed through the representation of the time series occurrence frequencies, using a probabilistic distribution $f(V)$, according to (1) 34]:

$$
\frac{\partial F(V)}{\partial V}=f(V)
$$

where the probabilistic distribution expression, the mean wind speed $(\bar{v})$ and the variance $\left(\sigma^{2}\right)$ are obtained through (2) and (3), respectively:

$$
\begin{gathered}
\bar{v}=\int_{0}^{\infty} v f(v) d v \\
\sigma^{2}=\int_{0}^{\infty}(v-\bar{v}) f(v) d v
\end{gathered}
$$

The CDF should represent the histogram of the simulated wind regime. There are several CDFs that are used to model wind speed, but the most commonly used is the Weibull CDF, whose mathematical model is described by (4) 35:

$$
f(v)=\frac{k(i)}{c(i)}\left(\frac{v}{c(i)}\right)^{k-1} e^{\left(-\frac{v}{c(i)}\right)^{k}},(K>0, v>0, c>1)
$$


where $v, k$ and $c$ are the wind speed $(\mathrm{m} / \mathrm{s})$, the form factor and the scaling factor, respectively.

To determine the form factor value, an acceptable approximation is given in [35] as (5):

$$
k(i)=\frac{\sigma(i)^{-1,086}}{\mu(i)},(1 \leq i \leq 96)
$$

where $\mu(i)$ and $\sigma(i)$ are the mean and standard deviation of the wind speed in the range $i$, respectively.

Once the form factor has been determined, the scaling factor can be determined through (6):

$$
c(i)=\frac{\sigma(i)}{\Gamma\left(1+\frac{1}{k(i)}\right)},(1 \leq i \leq 96)
$$

where $\Gamma$ is the Gamma function.

As for the solar irradiation modeling, the beta distribution is widely used in the literature for this purpose. As this distribution requires the variables to be in the range of [0-1], then the solar irradiance data should be normalized, as in (7) 36].

$$
f(r)=\frac{\Gamma(\alpha(i)+\beta(i))}{\Gamma(\alpha(i)) \times \Gamma(\beta(i))}\left(\frac{r}{r_{\max }(i)}\right)^{\alpha-1}\left(1-\frac{r}{r_{\max }(i)}\right)^{\beta-1}, \alpha>0, \beta>0,
$$

where $r$ is the solar irradiation $\left(k W / m^{2}\right), r_{\max }(i)$ the maximum irradiation in the period $i\left(k W / m^{2}\right)$, and $\alpha(i)$ and $\beta(i)$ the shape parameters in the period $i$. With the average $\mu(i)$ and the standard deviation $\sigma(i)$ of solar irradiation, it is possible to determine the parameters in the form $\beta(i)$ and $\alpha(i)$ of the beta distribution, through (8) and (9), respectively:

$$
\beta(i)=(1-\mu(i)) \times\left(\frac{\mu(i)(1-\mu(i))}{\sigma(i)^{2}}-1\right)
$$




$$
\alpha(i)=\frac{\mu(i) \beta(i)}{1-\mu(i)}
$$

In this work, $96 \mathrm{CDF}$ of Weibull and $96 \mathrm{CDF}$ Beta are generated to model the wind velocity and the solar irradiation, respectively. Each CDF represents one hour of the day of the four seasons.

\subsection{Stochastic Dependence Modeling}

In the wind and solar energy generation process, there is a stochastic dependence between the different random variables, the sources that generate such energies. To determine the correlation between wind velocity and solar radiation, the model used by 33 . is used as reference. To measure the dependency strength among random variables, the correlation rank $\left(\rho_{r}\right)$ is used. This rank has two random variables $X, Y$ and can be obtained by (10):

$$
\rho(X, Y)=\rho\left(F_{X}(X), F_{Y}(Y)\right)
$$

where $\rho\left(F_{X}(X), F_{Y}(Y)\right)$ is the Pearson correlation $(\rho)$ between two FDC $\left(F_{X}(X)\right.$ and $\left.F_{Y}(Y)\right)$, obtained from (11):

$$
\rho\left(F_{X}(X), F_{Y}(Y)\right)=\frac{\operatorname{Cov}\left(F_{X}, F_{Y}\right)}{\sigma\left(F_{X}\right) \sigma\left(F_{Y}\right)}
$$

where $C o v$ and $\sigma$ are the covariance and standard deviation, respectively. The correlation rank is symmetric and assumes values in the interval $[-1,1]$, where the negative end means that there is an inversely proportional correlation and the positive end a directly proportional correlation. The variables are completely independent when the correlation rank is zero. For uniform variables, the Pearson correlation is equal to the correlation rank $\left(\rho=\rho_{r}\right)$, but in most cases they are different. For normal multivariate distributions, the relation between $\rho$ and $\rho_{r}$ is shown in $(12)$ :

$$
\rho(X, Y)=2 \sin \left(\frac{\pi}{6} \rho_{r}(X, Y)\right)
$$

where $X$ and $Y$ are random vectors with normal multivariate distribution.

By using the $F_{X}(X)$ and $F_{Y}(Y)$, we deal with the rank of the distributions and no longer with exact values. With these ranks, it is possible 
to model the functional dependence of $X$ and $Y$. In order to investigate the properties of these distributions, the notion of copula for the modeling of stochastic dependence in the uncertainty analysis is used. The notion of copula was introduced to separate the effect of the dependence of the marginal distributions on a joint distribution, formulating multivariate distributions so that any dependency can be represented. Copulation is the joint cumulative distribution in a unit cube, such that the marginal distributions are uniform in the interval $[0,1]$. The random variables $X$ and $Y$ can be joined by copula $C$ if their joint distribution can be written by (13):

$$
F_{X Y}(X, Y)=C\left(F_{X}(X), F_{Y}(Y)\right)
$$

By definition, if $\operatorname{CDF} F_{X}(X)$ and $F_{Y}(Y)$ are invertible, then they can be written as $F_{X}(X)=u$ and $F_{Y}(Y)=v$, according to relation (14):

$$
X=F_{X}^{-1}(U) \Leftrightarrow U=F_{X}(X)
$$

where $u$ and $v$ are realizations for the uniform random variables $U$ and $V$ and can be written as (15):

$$
C_{V \mid U}(u, v)=F(X, Y)=F\left(F_{X}^{-1}(u), F_{Y}^{-1}(v)\right)
$$

where $C_{V \mid U}$ is the conditional distribution of $V \mid U(X$ and $Y$ copula) and the inverse of the standard univariate normal distribution function.

In the literature, there are many families of copulas. However, the proposed methodology uses the diagonal band copula (DBC) to model the stochastic dependence of wind speed with solar irradiation. DBC is selected as it is easily calculated and does not require numerical approximations, which makes it attractive in modeling correlated events. The DBC representation is shown in Figure 2 .

The DBC has a vertical bandwidth of $1-\theta$, where $\theta \epsilon[0,1]$. According to its width, the variables can be directly correlated $(\theta=1)$, inversely correlated $(\theta=-1)$ or independent $(\theta=0)$. Using this copula, it is possible to calculate the reverse conditional distribution: 


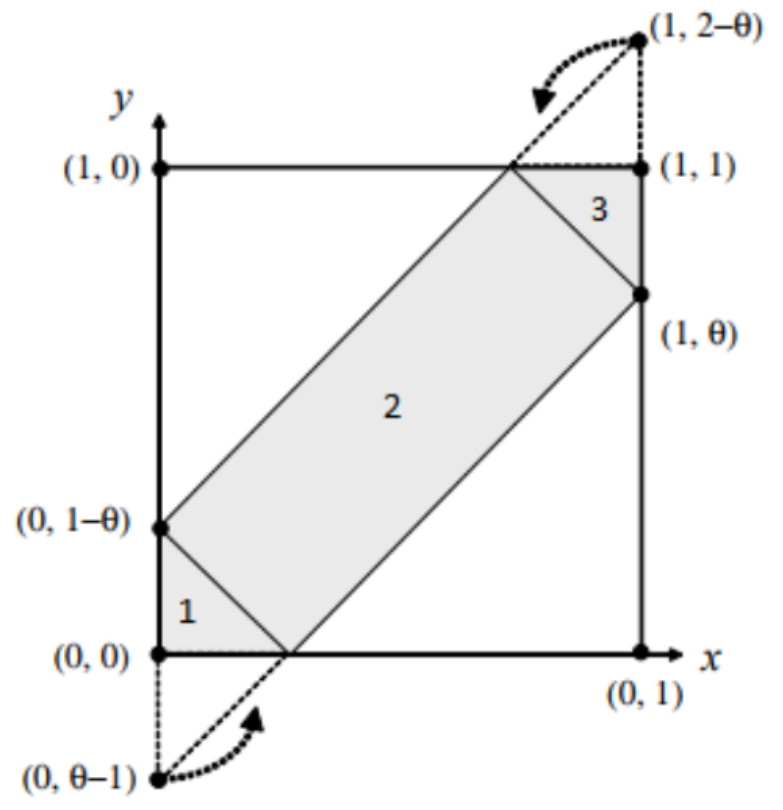

Figure 2: Diagonal band copula.

$$
C_{V, U}^{-1}(t \mid u, \theta)=\left\{\begin{array}{c}
(1-\theta) t, u<1-\theta, t<1-\frac{u}{1-\theta} \\
(1-\theta) t+\theta, u>\theta, t>\frac{1-u}{1-\theta} \\
2(1-\theta) t+u-1+\theta, \text { others }
\end{array}\right.
$$

In order to obtain the correlation of wind velocity with solar irradiance using DBC, the following algorithm is followed:

\section{Algorithm 2: Diagonal Band Copula.}

- Step 1) Starts with the first hour $(h=1)$ of $96 h$;

- Step 2) Two independent vectors are generated $\left(U_{1}\right.$ and $\left.U_{2}\right)$, each with 10,000 randomly distributed values in the interval $[0,1]$. The embodiments $u_{1}$ and $u_{2}$ are defined by (14);

- Step 3) The correlation rank between Weibull's CDF $\left(\left(F_{X}(X)\right)\right.$ and Beta $\left(F_{Y}(Y)\right)$ is calculated through $(10)$;

- Step 4) The Pearson coefficient of the wind speed and solar irradiation are calculated, using (12); 
- Step 5) $\theta$ is calculated for the CDF Weibull and Beta correlation, using (16);

- Step 6) The simulation of the correlation of random variables with the DBC can be done through (17), where $C_{V \mid U}^{-1}$ is obtained in $(16)$ :

$$
\begin{gathered}
u=u_{1} \\
v=C_{V \mid U}^{-1}\left(u_{2} \mid u, \theta\right)
\end{gathered}
$$

- Step 7) The original probabilistic density functions (PDF) of the wind speed and solar radiation are obtained through the inverse of the CDF in 10 .

- Step 8) $h=h+1$;.

- Step 9) While $(h \leq 96)$, go back to step (2). Otherwise, the algorithm finishes.

To obtain the annual correlation (divided into 96h), the algorithm must be repeated for the next hour until the $96 \mathrm{~h}$ of the season is completed.

\subsection{Wind Power Generation}

There are several methods in the literature to model the power of wind generators, among which we can mention the models based on the equations of energy available in the wind and the models based on the power curve of the wind turbine. Better precision in modeling is obtained with the latter, since the power curve directly provides the power generated for a specific wind speed [37]. Most small wind turbines use stall control to operate at maximum capacity, due to its simplicity and low cost. The machines that operate with the stall control have four operating modes, described below:

- The wind speed is less than the turbine cut-in speed $\left(V_{i}\right)$ : the wind power is not sufficient, therefore the turbine does not start and there is no generation of power.

- The wind speed is greater than $V_{i}$, but less than the nominal speed $\left(V_{n}\right)$ : this activates the power control system of the machine by adjusting the aerodynamic stall in order to work with the maximum allowed power. 
- The wind speed is between $V_{n}$ and the cut out speed $\left(V_{o}\right)$ : the power control system of the machine acts through the aerodynamic stall in order to work with the nominal power, preventing the machine to operate above the rated power.

- The wind speed is greater than $V_{o}$ : the turbine is turned off in order to avoid damage to its physical structure.

Based on the technical specifications and power data generated as a function of wind speed obtained from the manufacturer's manual, this work uses the least squares method to model wind power, whose mathematical model is described in 18, where $w$ is the wind speed and $a, b, c, d, e, f, g, h$, $i, j$ are the ninth degree polynomial coefficients, which are determined using the polyfit tool of the Matlab software [38]:

$$
P_{w}=\left\{\begin{array}{c}
0 ; 0 \leq V<V_{i} \\
a * V^{9}+\ldots+i * V+j ; V_{i} \leq V<V_{0} \\
0 ; V \geq V_{0}
\end{array}\right.
$$

where:

$V$ is the wind speed $(\mathrm{m} / \mathrm{s})$;

$V_{i}$ is the cut-in speed $(\mathrm{m} / \mathrm{s})$;

$V_{0}$ is the cut-out speed $(\mathrm{m} / \mathrm{s})$;

$a, \ldots, j$ are the coefficients of the polynomial.

$P_{w}$ is the power generated by wind $(\mathrm{W})$.

\subsection{Photovoltaic Power Generation}

The output power of photovoltaic panels depends on solar radiation, the temperature of the location and the physical characteristics of the photovoltaic module. The methodology proposed in [39] was used to model it. The mathematical model is presented in (19):

$$
\begin{gathered}
T_{c}=T_{a}+r\left(\frac{N_{o t}-20}{0,8}\right) \\
I=r\left[I_{s c}+K_{i}\left(T_{c}-25\right)\right] \\
V=V_{o c}-K_{v}\left(T_{c}-25\right) \\
F F=\frac{V_{m p p t} \cdot I_{m p p t}}{V_{c} \cdot I_{s c}} \\
P_{p h}=\frac{N * F * V}{I}
\end{gathered}
$$


where:

$T_{c}$ - photovoltaic cell temperature $\left({ }^{\circ} \mathrm{C}\right)$;

$T_{a}$ - average ambient temperature in the period $\left({ }^{\circ} \mathrm{C}\right)$;

$N_{\text {ot }}$ - nominal cell operating temperature $\left({ }^{\circ} \mathrm{C}\right)$;

$I$ - current in cell (A);

$I_{s c}$ - cell short circuit current $(\mathrm{A})$;

$K_{i}$ - current/temperature coefficient $\left(\mathrm{A} /{ }^{\circ} \mathrm{C}\right)$;

$V$ - voltage in the photovoltaic module $(\mathrm{V})$;

$V_{o c}$ - open circuit voltage of the module $(\mathrm{V})$;

$K_{v}$ - stress/temperature coefficient $\left(\mathrm{V} /{ }^{\circ} \mathrm{C}\right)$;

$F F$ - form factor (dimensionless);

$V_{m p p t}$ - voltage at the point of maximum power $(\mathrm{V})$;

$I_{m p p t}$ - current at the point of maximum power (A);

$N$ - Number of panels;

$P_{p h}$ - power generated by the panels $(\mathrm{W})$.

\subsection{Determining the Power of $D G$}

To determine the power supplied by the DG, preserving their stochastic nature, the Monte Carlo Simulation (MCS) is used, as it uses the generation of random numbers to assign values to the investigated variables. This method can be applied to both deterministic and stochastic problems. The Monte Carlo method is a numerical procedure that uses random, or pseudo-random, numbers to treat quantities that are not necessarily random, based on the Law of Large Numbers and the Central Limit Theorem. The technique consists in generating random values for each probability distribution within a model, in order to simulate a relatively large number of scenarios. In MCS, the average value of the number of simulations $(N)$ is associated with the statistical error estimate $(\epsilon)$, given by (20) [40]:

$$
N=\frac{1}{4 \times \delta \times \epsilon^{2}}
$$

where $\delta$ is the sample variance, which can be determined according to the $(\zeta)$ confidence interval in 21 :

$$
\delta=1-\zeta
$$

To obtain an error of $5 \%$, with a confidence interval of $99 \%$, it is necessary to generate 10000 random numbers, that is, 10000 scenarios. The power value 
with the highest probability of occurrence, among the 10000 random numbers of wind speed and solar radiation, is obtained through (22). The convergence of the MCS is guaranteed with a very high number of simulations (10000 simulations):

$$
P_{o}=\frac{1}{N} \sum_{j=1}^{N} P(j)
$$

where $P_{o}$ is the power value with the highest probability of occurrence calculated for each hour of each station, $P(j)$ is the power of the DG for each iteration and $N$ is the number of iterations. In this work, MCS is applied 96 times (once for each hour of the four seasons) and receives the PDF for wind speed and solar radiation. At the end of the 10000 iterations, there is a PDF of the power of the DG. The simulation algorithm is shown below:

\section{Algorithm 3: Monte Carlo Simulation}

- Step 1) 10000 random numbers are generated;

- Step 2) The CDF of the wind speed and solar radiation is obtained;

- Step 3) The correlation between the incoming CDF through the DBC is determined;

- Step 4) The inverse CDF is obtained (returning to the original PDF values);

- Step 5) The power of wind generators and photovoltaic panels is determined through the mathematical modeling shown in section 3.4;

- Step 6) The PDF of the power of the DG with the highest probability of occurrence is obtained.

\section{Radial Load Flow Algorithm}

The load flow, or power flow, determines the state and distribution of the electrical network flows for a given load. In this problem, the network is represented by a set of algebraic equations and inequalities, meaning that the system model is static. To calculate the load flow, computational methods specifically developed to solve systems of algebraic equations and inequalities 
are used. To model the network feeder, the radial topology was chosen, since it is the most used type in electricity distribution networks.

To calculate the load flow of the feeders, the sum of the power method was used, adapted from [41. This method uses a simple solution process, presenting an excellent performance to solve load flow problems in radial feeders. The algorithm for obtaining the flow is shown below:

Algorithm 4: Radial Load Flow

- Step 1) The system parameters are defined, such as the identification of the substation voltage (reference node) and the tolerance for convergence $(\epsilon)$;

- Step 2) The voltage values of the nodes are defined as being equal to the reference voltage with a zero angle and the total losses of active and reactive power are considered to be null;

- Step 3) Upstream operation: the sum of the powers at each node (starting at the most extreme nodes and going up to the substation) is calculated, including the losses obtained in (23) and (24):

$$
\begin{aligned}
P_{k m p} & =r_{k m} \frac{P^{2}+Q^{2}}{V_{m}^{2}} \\
Q_{k m p} & =x_{k m} \frac{P^{2}+Q^{2}}{V_{m}^{2}}
\end{aligned}
$$

where $P_{k m p}$ and $Q_{k m p}$ are the active and reactive losses between the bars $k$ and $m, r_{k m}$ and $x_{k m}$ are the resistance and reactance of the $k m$, $P$ and $Q$ are the active and reactive powers of the load, and $V_{m}$ is the modulus of the voltage in the load.

- Step 4) Downstream operation: new voltage values are calculated on all system bars (starting at the substation and going to the extreme nodes) using (25);

$$
V_{m}^{4}+\left[2\left(r_{k m} P+x_{k m} Q\right)-V_{m}^{2}\right] V_{M}^{2}+\left(P^{2}+Q^{2}\right)\left(r_{k m}^{2}+x_{k m}^{2}\right)=0
$$

where $V_{k}$ and $V_{m}$ are the voltages of the $k$ and $m$ nodes, $r_{k m}$ and $x_{k m}$ are the resistance and reactance of the $\mathrm{km}$, and $P$ and $Q$ are the active and reactive powers of the load. 
- Step 5) The new values of the active and reactive losses are calculated using (23) and (24);

- Step 6) Convergence criteria: if the difference between the total losses of the current iteration and the previous iteration is less than the tolerance value for convergence, the process must be ended. Otherwise, go back to step (3).

\section{Problem formulation and the Proposed Genetic Algorithm Op- timization}

In this work, we opted to allocate the $\mathrm{CB}$ on the same bar in which the $\mathrm{DG}$ were installed. The presence of CB in the electrical network allows the surplus of the circuit reactive to be supplied, consequently increasing the power factor and the current of the feeder. As a result, there is an improvement in the voltage profile of the network, an increase in the supply capacity and a contribution to the reduction of system losses. The CBs used were those of commercial values, 600 kvar and 1200 kvar.

As input to the AG, there is a vector with 96 values of active power $(24 h \times 4$ seasons $)$ from the DG and, for each feeder, the heavy load level (higher load value) observed hour, considering the horizon of one year, as in [42]. The location of the DG is represented by a chromosome with $2 \times k$ bits, $k$ being the number of bars in the feeder. The encoding used is binary, where each pair of elements on the chromosome indicates whether there will be the installation of DG and CB units, so that 00,01,11, 10 mean, respectively, that there is no DG or CB; there is no DG, but there are CBs; there are DGs and $\mathrm{CBs}$; and there are DGs, but there is no CB.

When inserting a DG and a $\mathrm{CB}$ in a certain bar, it will be possible to supply the load of this bar and, when necessary, supply the surplus to the system. The necessary modifications to represent the presence of DG and CB are shown in (26) and (27), respectively.

$$
\begin{aligned}
& \Delta P_{k}=P_{D k}-D G_{k} \times P_{D G k} \\
& \Delta Q_{k}=Q_{D k}-C B_{k} \times Q_{C B k}
\end{aligned}
$$

where $\Delta P_{k}$ is the resulting active power variation $(W)$ in the $k$ bar, $P_{D k}$ is the active power $(W)$ demanded in the $k$ bar, $D G_{k}$ is the binary variable for 
determining the presence of $D G$ in the $k$ bar, $P_{D G k}$ is the active power $(W)$ injected by the $D G$ into the $k$ bar, $\Delta Q_{k}$ is the variation of reactive power (kvar) resulting in the bar $k, Q_{D k}$ is the reactive power (kvar) demanded in the bar $k, C B_{k}$ is the variable binary to determine the presence of $C B$ in the $k$ bar and $Q_{C B k}$ is the reactive power (kvar) injected by $C B$ into the $k$ bar.

\subsection{Mathematical Formulation of the Problem}

In practice, in addition to minimizing active power losses, it is also necessary to minimize the overall cost of the project. Although this problem

is multiobjective, a mono-objective formulation can be used that integrates the losses of active power and the global cost in a single function as follows:

Minimize:Annual active power losses and Global Cost

\section{$S . T$.}

Active and reactive power balance Current magnitude limit on EPS lines

Voltage magnitude limits at EPS nodes

Operational limit of $D G$

Quantitative limit of $D G$

In the proposal, we seek to minimize the annual active power losses of the feeders, as well as the overall cost of implementing the project. In this work, the costs related to the installation and maintenance of the DG and CB units are considered, in addition to respecting the maximum voltage limits on the bars as stipulated by the regulatory agency. For each hourly interval, the insertion of DG and CB must meet the restriction of the balance of active and reactive power in the EPS bars, according to (29) and (30), respectively:

$$
\begin{aligned}
& \Delta P_{k}=D G_{k} \times P_{D G}-P_{D k}-P_{k}(V, \theta)=0 \\
& \Delta Q_{k}=C B_{k} \times Q_{C B}-Q_{D k}-Q_{k}(V, \theta)=0
\end{aligned}
$$

The number of DG and CB units installed in the EPS is limited by the restrictions shown in 31 and $(32)$ :

$$
\sum_{k=2}^{n b} D G_{k}=N_{D G}
$$




$$
\sum_{k=2}^{n b} C B_{k} \leq N_{C B}
$$

where $D G_{k}$ is the binary variable for determining the presence of $D G$ in the $k$ bar, $C B_{k}$ is the binary variable for determining the presence of $C B$ in the $k$ bar, $N_{D G}$ is the maximum allowed number of $D G$ in the system and $N_{C B}$ is the maximum allowed number of $C B$ in the system.

\subsection{Objective Function}

The proposed GA uses an objective function $(O F)$ to minimize the global cost of installing the $D G, C B$ and annual losses of active power of the system and to meet the operating restrictions of the distribution network, as shown in 33 :

$$
O F=\operatorname{mim}\left[\left(\alpha_{1} C_{g}-\alpha_{2}\left(\sum_{i=1}^{96}\left(\Delta P_{l}^{i}+P_{D G}^{i}\right)\right) \times t_{e}\right)+\beta \sum_{i=1}^{96}\left(\sum_{k=1}^{b n}\left|\Delta V^{k}\right|\right)_{i}\right]
$$

where $\Delta P_{l}^{i}$ represents the reduction of active power losses in the hourly interval $i$ in relation to the original feeder losses before the insertion of DG and $\mathrm{CB}, P_{D G}^{i}$ represents the active power generated by the $D G$ in the hourly interval $i, t_{e}$ is the tariff of the energy purchased by the concessionaire, $C_{g}$ represents the cost of installation and maintenance of the DG and CB and represents the deviations of tension in the $k$-th node of the network. The constants $\alpha_{1}, \alpha_{2}$ and $\beta$ are the penalties imposed on the terms of the objective function in order to obtain the best solution to the optimization problem.

A scheme of the GA integrated with the power flow to obtain the optimal allocation of the DG and CB units is shown in the subsection 4.3. In the evaluation step, each solution (power of an hourly interval) is subjected to the calculation of the power flow until the 96 hourly intervals are completed. Therefore, for each hourly interval, the total annual active power losses and the total cost of installation and maintenance of the DG and CB units are updated.

When calculating the power flow, the system bus voltages are also obtained. If any bar does not meet the voltage restrictions, or if the system does not have the maximum number of DG, then the individual is penalized, so that it has less chance of generating descendants in the next generation. 


\subsection{Binary Genetic Algorithm}

A genetic algorithm is a meta-heuristic inspired by the theory of evolution, in which the best individual is normally more likely to pass its genetic load on to future generations [43]. Through computation, the algorithm tries to recreate the necessary conditions to find a better individual after a certain number of iterations (generations).

The initial population of the GA is randomly generated and the genetic operators on the chromosomes are selection, crossing and mutation. Selection is the genetic operation in which the most suitable chromosomes are chosen to form the genetic basis for the subsequent generation. The crossing adopted in this work consists of drawing two individuals through the roulette method, where a cut is made in the chromosomes (individuals) drawn. From this, two new individuals are generated through an exchange of stretches of genes. The mutation operator is necessary for the introduction and maintenance of the genetic diversity of the population, arbitrarily altering one or more genes on a chromosome. The chromosome is represented by a vector that assumes 0 for possible positions when certain equipment is not considered and 1 for positions when it is intended to consider the equipment.

The GA performs the following steps:

\section{Algorithm 5: Genetic Algorithm}

- Step 1: Generate the initial random population and evaluate each solution obtained;

- Step 2: Draw the members of the population who will perform the crossing;

- Step 3: The solutions chosen in the previous step will be recombined given a probability of crossing;

- Step 4: The mutation is applied to all members of the population limited by a probability;

- Step 5: Choose the elite solution and place it in the current population, avoiding the loss of the best solution from the previous generation;

- Step 6: Evaluate the solutions obtained using the objective function using (33); 
- Step 7: If the specified number of generations is reached, then the algorithm finishes. Otherwise, return to step (2).

\section{Results}

In this work, to demonstrate the proposal, two real radial feeders from Light Serviços de Eletricidade S.A are used: the one corresponding to the distribution line Bandeira, which has 78 bus and the one corresponding to the distribution line Recife, with 96 bus, located in Rio de Janeiro city (22 54 '23" south latitude and $43^{\circ} 10^{\prime} 21^{\prime \prime}$ west longitude), Brazil [42]. The methodology proposed in this work was implemented in Matlabß and the results of each stage of the methodology are now presented.

\subsection{Meteorological and load historical series}

To model power generation, data from the historical series of wind speed, solar irradiance and temperature were used in the period between 2010 and 2014, every 1 hour, from a meteorological station close to the region of study. The data of wind speed, solar irradiance and temperature varied according to the time of day and month of the year, so, its seasonality must be preserved. Each season of the year was subdivided into 24 hour segments (hour of day), with each hour representing a specific time for the entire season. Therefore, the year is divided into 96 time segments ( $24 \mathrm{~h} \times 4$ stations). Considering 30 days a month, each time segment had 450 data readings ( 5 years $\times 30$ days / month $\times 3$ months / station $\times 1$ read / hour). With this division, it was possible to preserve the seasonality characteristics in the generation.

The mean and standard deviation of each hourly interval of solar irradiation and wind speed are needed to obtain its probabilistic model from the Beta and Weibull distributions, respectively. The annual mean values of solar irradiation for each time interval are shown in 3.(a), and the same for wind speed in 3.(b).

According to Figure 3. (a), as expected, there is negligible solar radiation between approximately $7 \mathrm{pm}$ and $5 \mathrm{am}$, so, there is no generation of solar energy in this period. It is also noted that the peak irradiation occurs around noon, being higher in summer than in other seasons. In turn, according to Figure 3.(b), there is wind throughout the day, and the wind speed is higher at the end of the day, between $4 \mathrm{pm}$ and $8 \mathrm{pm}$. Furthermore, summer and spring were the seasons in which the highest wind averages occurred throughout the year. 
(a)

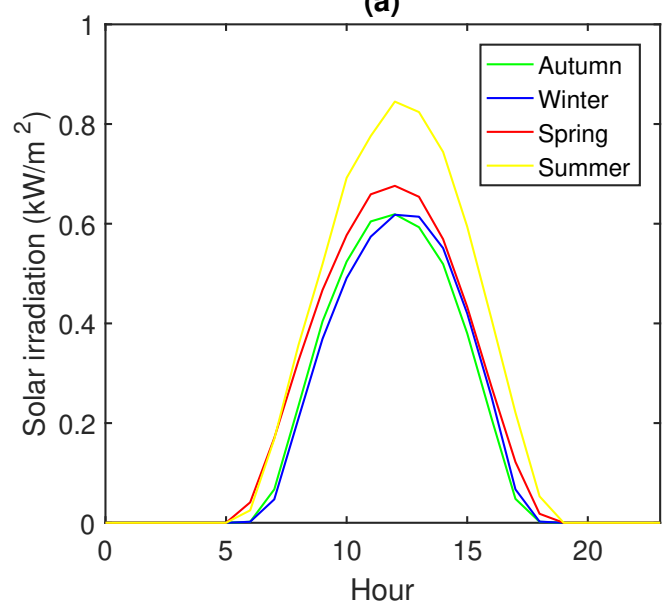

(b)

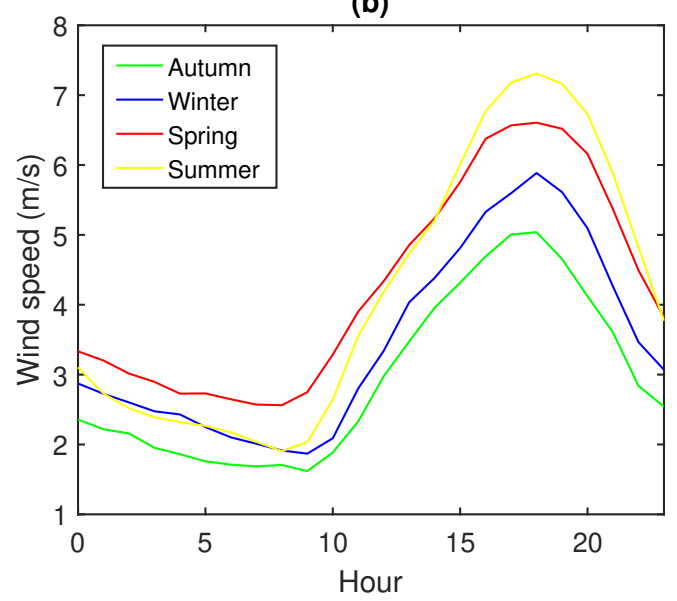

Figure 3: Historical series of a) solar irradiation b) wind speed, in 96 hourly intervals.

\subsection{Monte Carlo simulation}

In this subsection, the results obtained with the proposed methodology to model wind and solar generation are presented, considering their horoseasonal nature and stochastic correlation.

\subsubsection{Wind Speed PDF Modeling}

With the scale factor and shape values of the Weibull distribution obtained from a specific time interval, a Monte Carlo Simulation was performed with 10,000 iterations, in order to generate 10,000 random wind speed values, chosen randomly in the PDF of that time interval. The PDF of the wind speed of a time interval obtained by the Monte Carlo simulation described above is shown in Figure 4 and compared to the PDF of the real time series data.

\subsubsection{Wind Power Generation}

In this validation, a group of 60 small wind generators is considered, which corresponds to a total installed power of $600 \mathrm{~kW}$. To model the power generated by the generator when operating between the starting and cutting speed range, a 9 degree polynomial is used, whose coefficients were determined, using the least squares method, from the technical data provided by the manufacturer. The modeling of the turbine power with the 9 degree polynomial obtained a coefficient of determination $\left(R^{2}\right)$ of 0.9998 , proving the precision of the model 


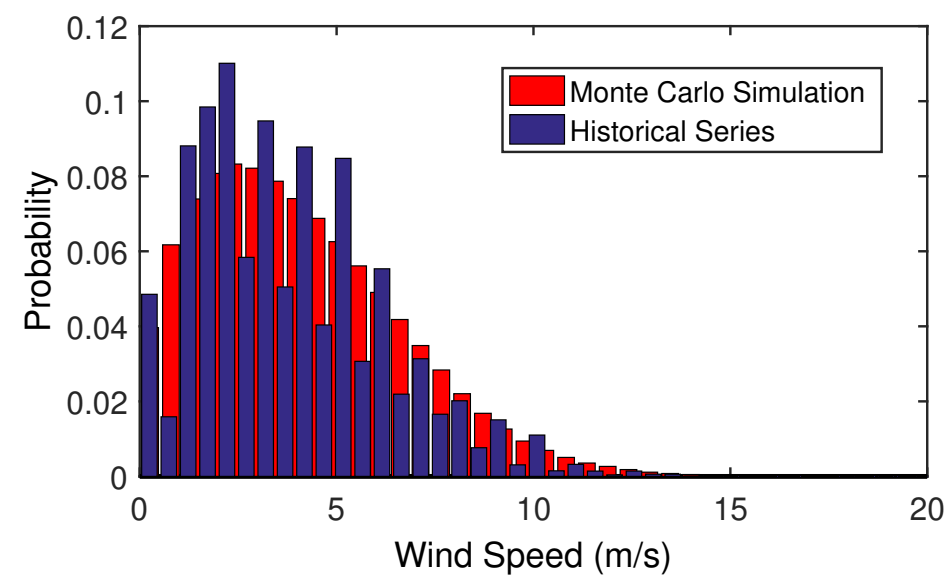

Figure 4: Annual PDF generated by Monte Carlo Simulation, and historical series for wind speed.

to describe the phenomenon. The curve obtained with the adopted model and the curve generated by the data in the manufacturer's manual are shown in Figure 5 .

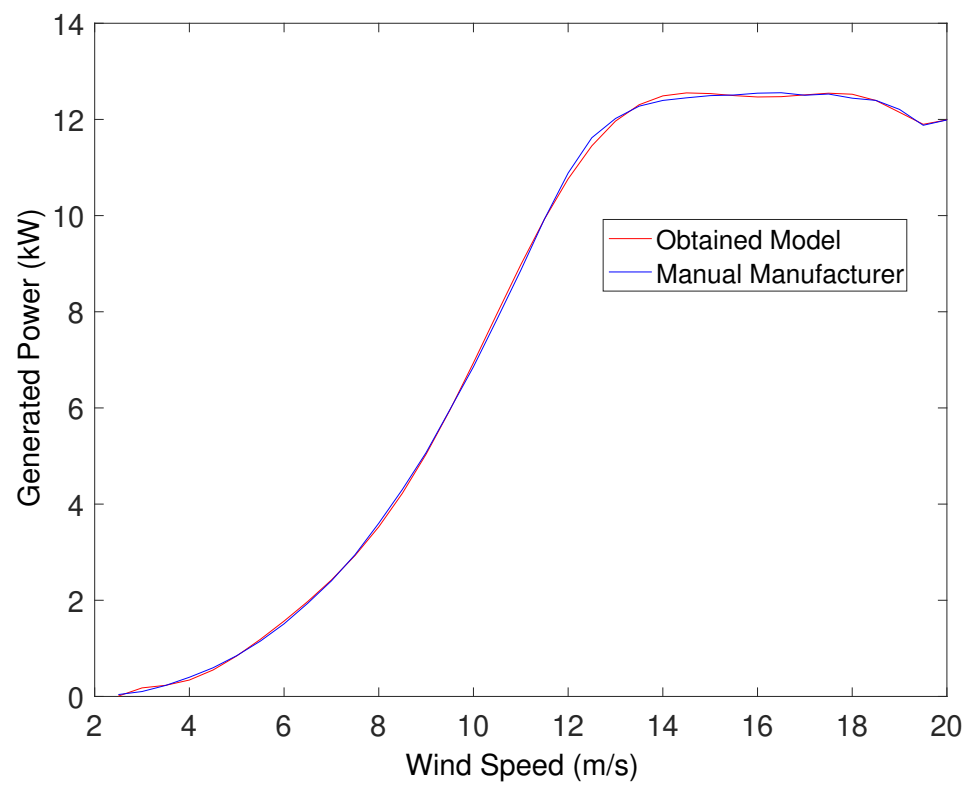

Figure 5: Power curves from the proposed model and the manufacturer's manual. 
Using the 9 degree polynomial, the power generated by the turbine as a function of wind speed is obtained for the 10,000 random wind speed numbers, considering each 96 PDF hourly interval. To obtain the total power of the generator group, it is necessary to multiply the power generated by the number of generators (60 generators). Figure 6 shows the PDF of power generated by the wind generators corresponding to 15 hours of autumn.

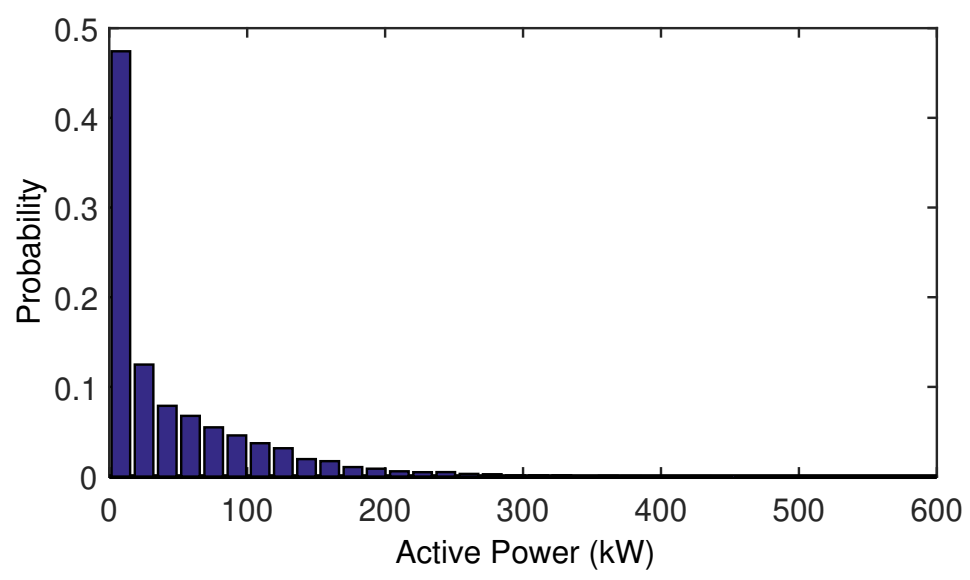

Figure 6: PDF of the active power generated from wind in fifteen hourly intervals during autumn.

As it can be seen in Figure 6, 48\% of the time the wind turbine is switched off in this time interval; this is because the wind speed is smaller than the cut-in speed of the machine $(2.5 \mathrm{~m} / \mathrm{s})$; the same percentage of time, the study region does not have a great wind potential.

\subsubsection{Solar Irradiation PDF Modeling}

As with the modeling of the wind speed profile, for each time interval, the respective solar irradiation profile was modeled using the beta distribution, which uses its mean and deviation values to obtain the $\alpha(i)$ and $\beta(i)$ shape factors. Thus, the PDF of one of the 96 intervals is created by the 10,000 solar irradiation values generated by the MCS. Figure 7 shows the solar irradiation PDF generated by the MCS and the respective PDF of the real historical series corresponding to 15 hours of autumn.

\subsubsection{Photovoltaic Generation}

In this study, a group of 6000 photovoltaic modules is considered, which corresponds to a total installed power of $1500 \mathrm{kWp}$. To model the power 


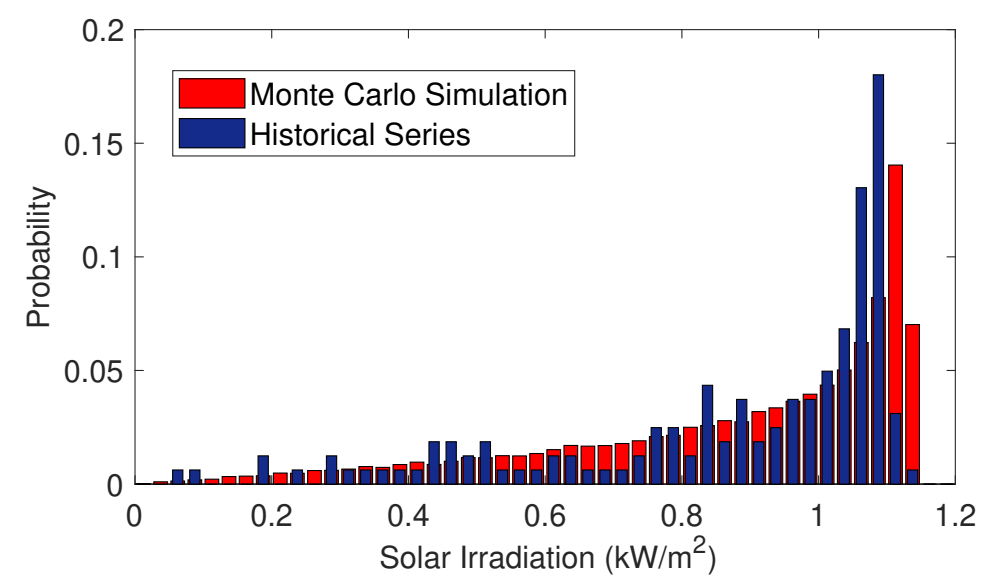

Figure 7: Annual PDF generated by MCS, and the historical series of solar irradiation.

generated by the panels, the methodology described in section 2.4 was used. Figure 8 shows the PDF of power generated by the solar panels corresponding to 15 hours of autumn.

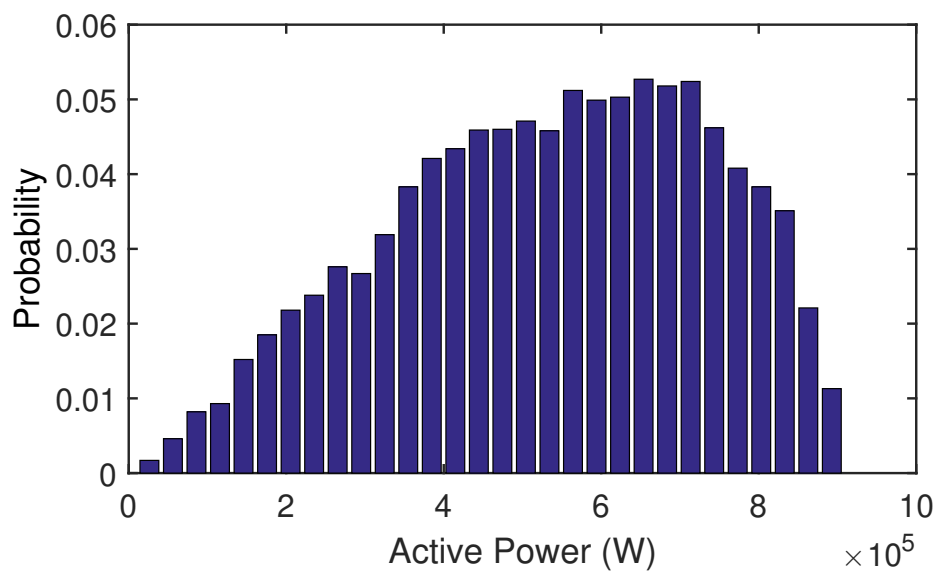

Figure 8: PDF of the active power generated by PVs in fifteen hourly intervals during autumn.

\subsubsection{Stochastic correlation of variables}

To model the stochastic dependence between the variables of wind speed, solar radiation, ambient temperature and load, the Diagonal Band Copula 
method was used, correlating each of the quantities with the wind speed and the load with the ambient temperature. Figure 9 shows the correlation rank among the listed variables.

The correlation rank informs the degree of correlation between the quantities, ranging from independent when equal to 0 , to strongly dependent directly when equal to 1 , or strongly dependent inversely when equal to -1 . It can be observed that there is a predominance of weak to moderate direct dependence among the quantities, since the maximum correlation modulus is always less than 0.5. The highest dependence values were found near the 12 th hour and it is positive (i.e., dependent directly) when analyzing the wind speed versus solar radiation, and wind speed versus temperature.

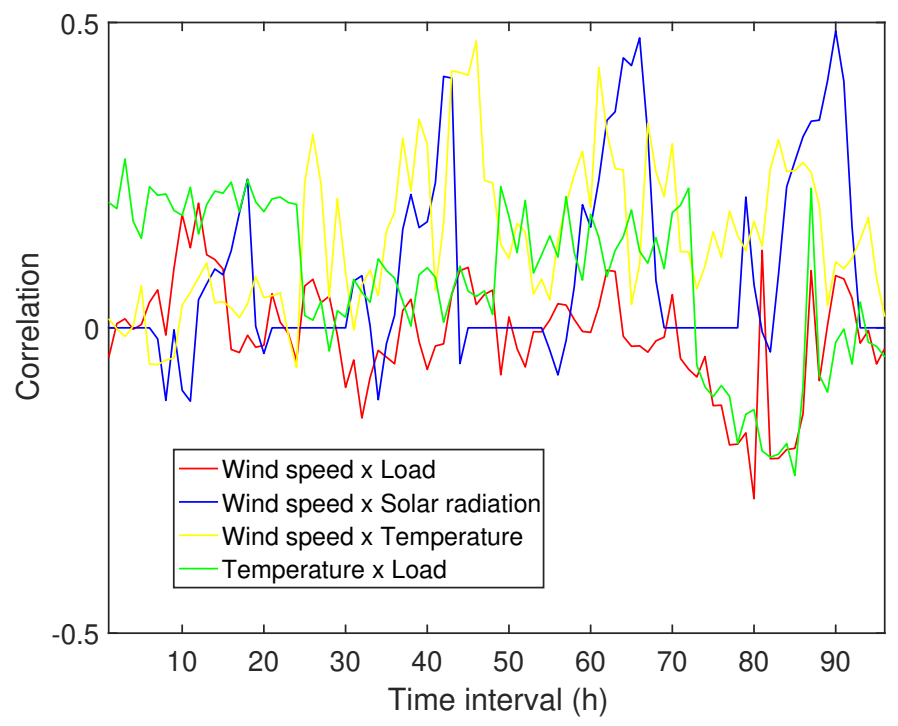

Figure 9: Correlation of the load with meteorological data.

\subsubsection{Total Power of $D G$}

The total power of the DG corresponds to the sum of the power of the wind generation with the power of the photovoltaic generation, obtained through the integration of the sequential Monte Carlo method and diagonal band Copula. The annual power generation, considering and not considering, the stochastic dependence modeling can be seen in Figure 10.

Analyzing Figure 10, it is clear that the photovoltaic power has more impact than the wind power because, in addition to having greater installed 

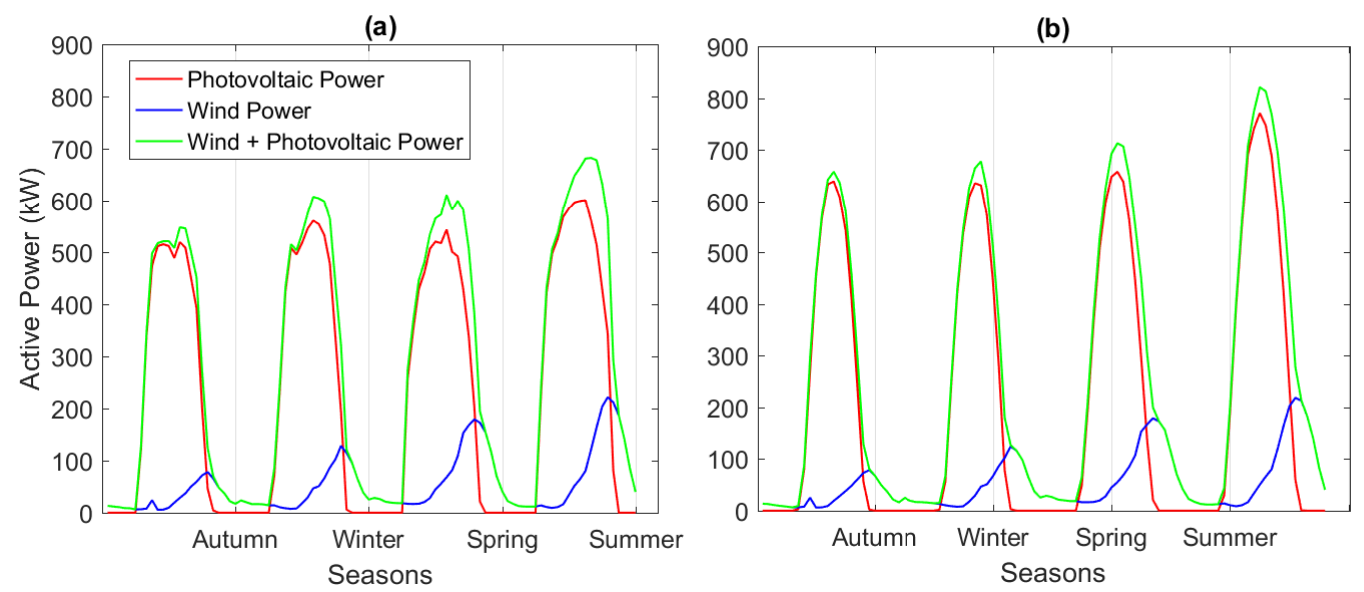

Figure 10: Results of annual power generation by DG a) using stochastic dependence modeling and b) without the model

capacity, the solar potential is better than the wind potential in the study region. It is also worth noting that the power generated by the DG (solar + wind), considering the correlation model is lower close to midday (at peak generation), but higher in the late afternoon (between 4 and $7 \mathrm{pm}$ ), because it has the highest positive Pearson coefficient of the wind speed and solar irradiation at these moments.

\subsection{Optimal Allocation of $D G$}

In this work, GA is used as a method of meta-heuristic optimization to search for the ideal installation location for the DG and CB units, with the premise of minimizing the overall cost of installing them and minimizing the annual loss of active power. To analyze the behavior of the proposed method, computer simulations were performed using the MATLAB software. The software and hardware used have the following configurations: Asus K45A-VX282H Notebook, 2.5 GHz Intel Core i5-3210M Processor, 4 GB RAM, MATLAB R2013a (8.1.0. 604).

Two radial systems were used: the 78-bus feeder from the Bandeira network and the 96-bus feeder from the Recife network. For each feeder, a total of five scenarios were considered for analysis: the base case, where no DG were connected to the system, with the allocation of five, four, three units and two DG.

The GA configuration was chosen from several tests and the parameters 
adopted were: Selection by roulette method, Crossing with a cutoff point, Crossover rate: $60 \%$, Mutation rate: $0.5 \%$, Size of the population: 40 individuals, Stopping criterion: maximum of 50 iterations (generations). The $\alpha_{1}, \alpha_{2}$ and $\beta$ constants of the GA objective function, as shown in (33), were defined from various tests and assumed the following values: $\alpha_{1}=0.000001, \alpha_{2}=10$ and $\beta=1000$.

The quantities used in this work are presented in a standardized way (Per Unit, p.u.), that is, based on predetermined base values. The basic values adopted for power and voltage are $1 M V A$ and $13.8 \mathrm{kV}$, respectively. The $\mathrm{AG}$ receives the $96 \mathrm{PDF}$ of power as input from the $\mathrm{DG}$, which represent the 24 hours of the day of the four seasons and the load levels of the feeders. The total annual power of the DG is approximately $33.90 \mathrm{pu}$, which is used as the total fixed amount generated by the DG, that is, for each scenario, the total installed power is $33.90 \mathrm{pu}$, and it can be divided equally into up to five generation units. The tests were used considering the worst case of load demand, that is, that with the highest hourly load.

From the feeder point of view of the voltage profile in steady state, the voltage in each feeder bus is classified as adequate, precarious and critical according to:

- Adequate: Voltage $>=0.93$ and $<=1.05$ p.u.;

- Precarious: Voltage $>=0.90$ and $<0.93$ p.u.;

- Critical: Voltage $<0.93$ or $>1.05$ p.u.;

Next, the results from the different test scenarios are presented and discussed.

\subsubsection{Base Case with no DG Connected to the System}

In this scenario, it is considered that no DG are connected to the system and that the values of the active power annual losses for the Bandeira (78 bus) and Recife (96 bus) feeders is approximately 44.70 p.u. and 37.17 p.u., respectively.

For this scenario, the voltage level of almost all the bus on both feeders is at a precarious or critical level, i.e., directly affecting the energy quality delivered to the consumer, while also generating a fine to the concessionaire for breach of voltage. The voltage profile of the 78 bus, when no DG are allocated, is shown (as a black line) in Figure 13. 


\subsubsection{Allocation of Five $D G$}

In this scenario, it is considered that five DG are connected to the system, together with the possibility of connecting up to five CBs to correct the critical voltage level in the feeder bus. Table 1 shows the optimal location of the bus for installing the DG and the value of the installed CB. The voltage profile of the 96 bus feeders, with the allocation of five DG, is shown in Figure 11 .

Table 1: Capacitor bank installed in the DG allocation bus.

\begin{tabular}{l|l|l|l}
\hline $\begin{array}{l}\text { Bus } \\
\text { Bandeira }\end{array}$ & $\begin{array}{l}\text { Power } \\
(\mathrm{kVAr})\end{array}$ & $\begin{array}{l}\text { Bus } \\
\text { Recife }\end{array}$ & $\begin{array}{l}\text { Power } \\
(\mathrm{kVAr})\end{array}$ \\
\hline \hline 16 & 1200 & 32 & 1200 \\
41 & 600 & 46 & 1200 \\
45 & - & 75 & 600 \\
60 & 600 & 88 & 600 \\
64 & 1200 & 89 & - \\
\hline
\end{tabular}

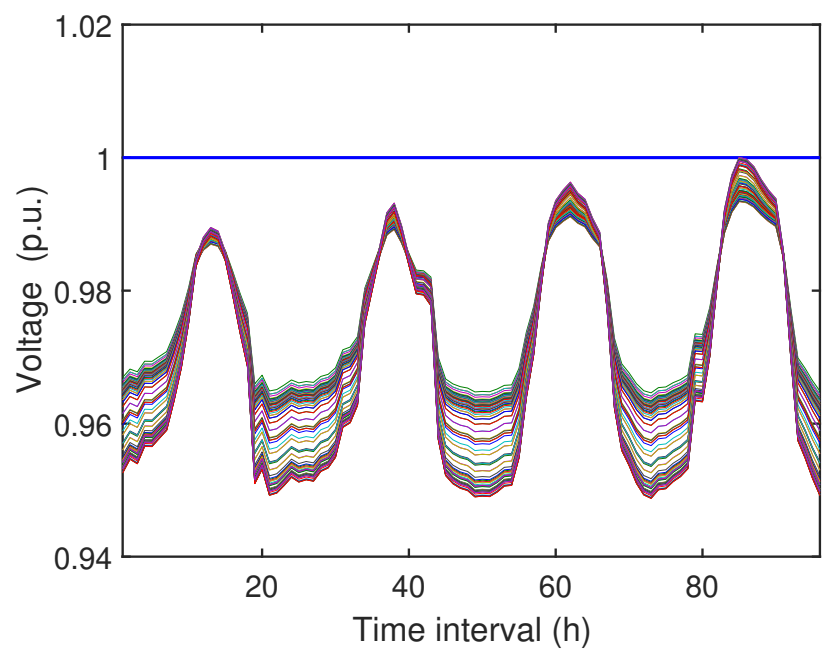

Figure 11: Annual voltage profile of the 96 bus feeder with allocation of 5 DG.

In the scenario with the allocation of five DG, according to Figures 11 , the voltage levels remain within the range considered adequate (between 0.93 and 1.05 p.u.) for all 96 hourly intervals of the 96 bus feeder. In both feeders, the best solution verified by the GA was the installation of four CBs. 


\subsubsection{Allocation of Four DG}

In this scenario, it is considered that four DG are connected to the system, together with the possibility of connecting up to four $\mathrm{CB}$ to correct the critical voltage level in the bus feeder. The voltage profile of the 78 bus feeder, with the allocation of four DG, is shown in Figure 12.

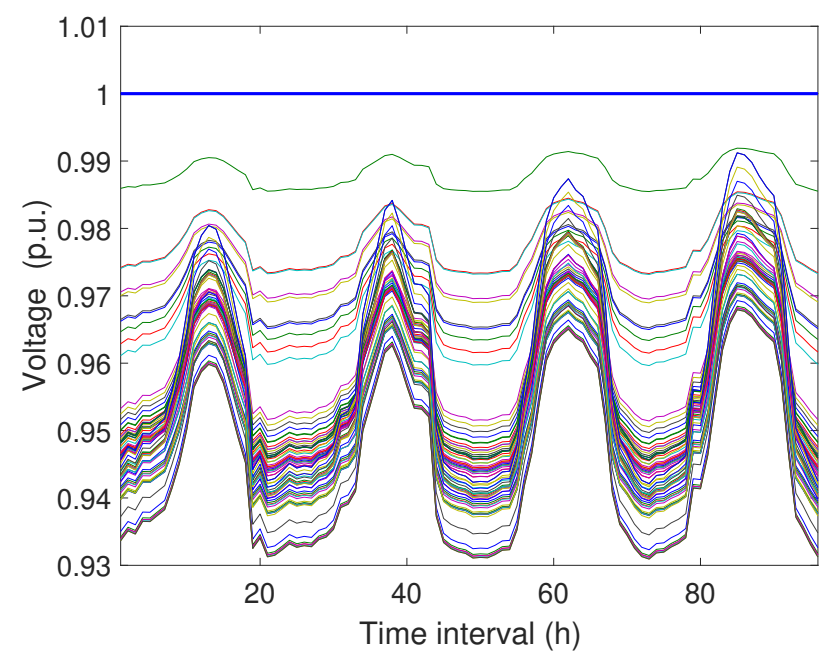

Figure 12: Annual voltage profile of the 78-bus feeder with allocation of 4 DG.

Table 2 shows the optimal location of the bus for installing the DG and the value of the $\mathrm{CBs}$, when installed.

Table 2: Capacitor bank installed in the DG allocation bus.

\begin{tabular}{l|l|l|l}
\hline $\begin{array}{l}\text { Bus } \\
\text { Bandeira }\end{array}$ & $\begin{array}{l}\text { Power } \\
(\mathrm{kVAr})\end{array}$ & $\begin{array}{l}\text { Bus } \\
\text { Recife }\end{array}$ & $\begin{array}{l}\text { Power } \\
(\mathrm{kVAr})\end{array}$ \\
\hline \hline 50 & 1200 & 32 & 1200 \\
52 & 1200 & 50 & 1200 \\
60 & - & 86 & 600 \\
78 & 600 & 94 & - \\
\hline
\end{tabular}

\subsubsection{Analysis of all scenarios}

By reducing the number of DG allocated, according to Figure 13, the voltage levels remain adequate; however, it is noticeable that there is an 
approximation to the lower limit of adequacy (0.93 p.u.). In both feeders, the best solution verified by the GA was the installation of three CBs.

In order to better visualize the relationship between the number of DG allocated and the voltage level of the bus feeder, Figure 13 shows, respectively, the voltage profile in the 78 bus feeder and in the 96 bus feeder at $3 \mathrm{pm}$ in the autumn for the different proposed scenarios.

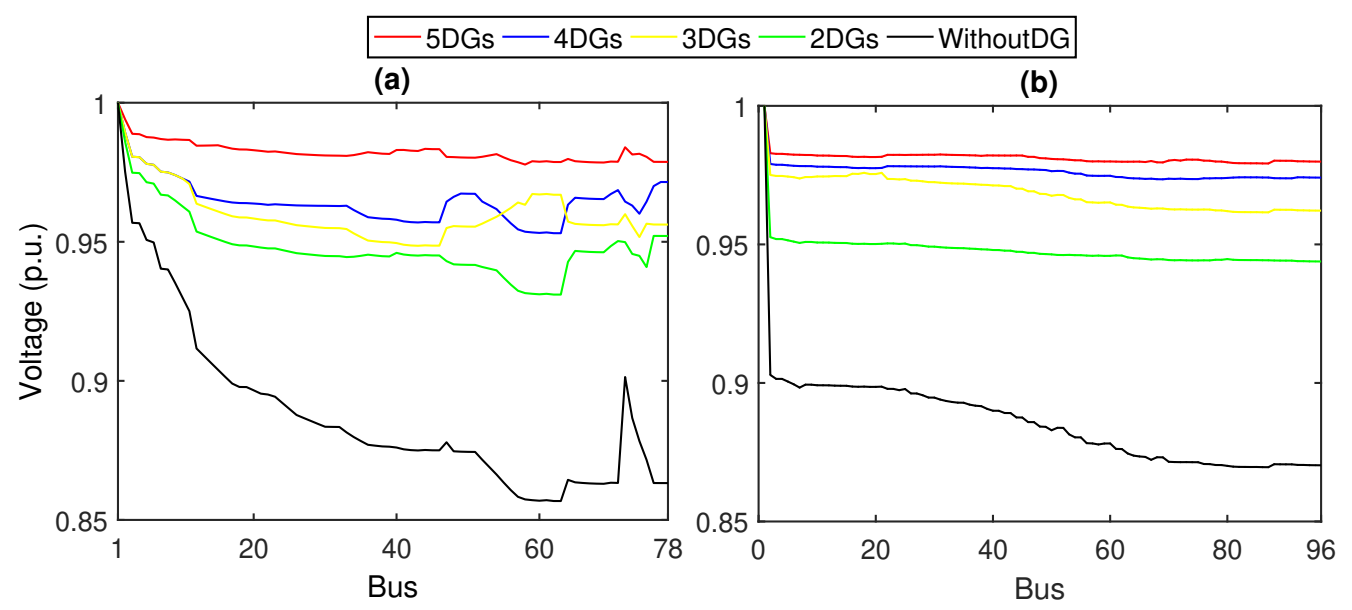

Figure 13: Voltage profile on the a) 78 bus and b) 96 Bus feeder at 3 pm in the autumn.

According to Figure 13, it can be observed that the voltage profile in both feeders increases as more DG are installed in the system. For the hourly interval of $3 \mathrm{pm}$ in the autumn, it appears that, even in the scenario with two DG allocated, the voltage profile remains at adequate levels. This fact is due to the low demand for load in this hourly interval, in contrast to other intervals where the demand is greater and the tension reaches precarious levels for this same scenario.

To perform a voltage profile analysis across all 96 time intervals, the Table 3 shows the number of bus from the Bandeira and Recife feeders that are in the precarious or inadequate range in at least one of the 96 intervals.

According to Table 3 , it can be observed that most bus are at the critical voltage level for at least one time interval, in both feeders, in the base case (without DG). In the case of 2 DG installed, the voltage profile improves, as it does not have bars with voltage at a critical level, but the Bandeira feeder still has 56 bus with voltage at a precarious level, while the Recife feeder has 9 bars in this situation. In scenarios with three or more DGs installed, all 
Table 3: Number of bus from the feeders that are in precarious or inadequate range in at least one of the 96 intervals.

\begin{tabular}{c|c|c|c|c}
\hline \multicolumn{2}{c|}{ Scenario } & \multicolumn{3}{c}{ Voltage bus profile (unit) } \\
\cline { 3 - 5 } \multicolumn{1}{c|}{} & Adequate & Precarious & Critical \\
\hline \hline \multirow{5}{*}{ Bandeira (78 bus) } & Base case & 10 & 7 & 61 \\
& $2 \mathrm{DG}$ & 26 & 56 & 0 \\
& $3 \mathrm{DG}$ & 96 & 0 & 0 \\
& $4 \mathrm{DG}$ & 96 & 0 & 0 \\
& $5 \mathrm{DG}$ & 96 & 0 & 0 \\
\hline \hline \multirow{5}{*}{ Recife (96 bus) } & Base case & 1 & 3 & 92 \\
& $2 \mathrm{DG}$ & 87 & 9 & 0 \\
& $3 \mathrm{DG}$ & 78 & 0 & 0 \\
& $4 \mathrm{DG}$ & 78 & 0 & 0 \\
& $5 \mathrm{DG}$ & 78 & 0 & 0 \\
\hline
\end{tabular}

buses are always within the critical voltage range, that is, the feeder voltage profile is within desirable standards.

\subsubsection{Annual losses of active power in the feeders}

Table 4 shows the annual losses of active power in the Bandeira and Recife feeders in the different test scenarios for installing the DG. It can be seen in Figure 14 that, for both feeders, the insertion of DG together with the CBs minimized annual losses significantly. There was a reduction of up to $71.7 \%$ in annual losses of active power in the Bandeira feeder and $73.4 \%$ in the Recife feeder in the best case (5 DG installed).

Table 4: Comparison of active power losses for different allocation scenarios of the DG.

\begin{tabular}{c|c|c}
\hline Scenario & $\begin{array}{c}\text { Annual losses (p.u.) } \\
\text { Bandeira }\end{array}$ & $\begin{array}{c}\text { Annual losses (p.u.) } \\
\text { Recife }\end{array}$ \\
\hline \hline Base case & 44.70 & 37.17 \\
5 DG & 12.65 & 9.87 \\
4 DG & 18.37 & 11.26 \\
3 DG & 20.02 & 13.67 \\
2 DG & 23.09 & 17.02 \\
\hline
\end{tabular}

Analogous to the voltage profile, the active power losses also increase with the number of installed DG, as shown in Figure 15. 


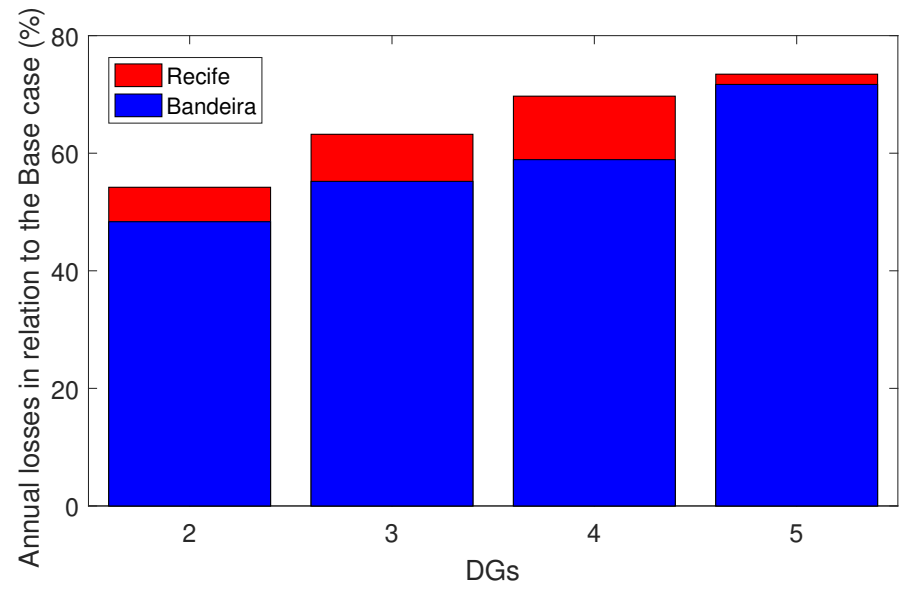

Figure 14: Annual active power losses, in relation to the Base case, of Bandeira (78-bus) and Recife (96-bus) feeders, with DG allocation.

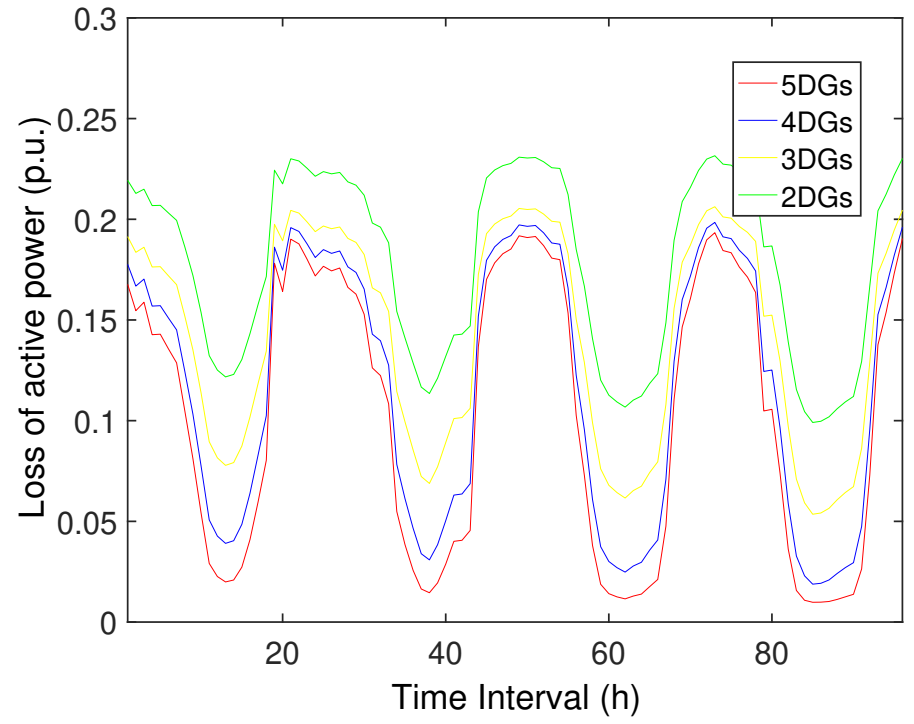

Figure 15: Profile of active power losses in the 96-bus feeder with DG allocation.

\subsubsection{Return on investment in feeders}

Tables 5 and 6 compare the investment needed, the annual maintenance costs, the annual revenue recovered and the Return on Investment (ROI) with the installation of the DG and CB units. The ROI is an expectation, 
considering the investment and maintenance costs and the revenue recovered annually by the DG and CB units installed in the network.

Table 5: Analysis of the Return on Investment (ROI) for the Bandeira feeder

\begin{tabular}{l|r|r|r|r}
\hline Scenario & Invest $(\mathrm{R} \$)$ & Maint.(R\$/yr) & Annual inc(R\$) & ROI (yrs) \\
\hline \hline Case 1 & $11,413,266$ & $142,806.60$ & $2,042,817.11$ & 6.08 \\
Case 2 & $11,408,626$ & $142,342.60$ & $1,865,651.69$ & 6.65 \\
Case 3 & $11,408,626$ & $142,342.60$ & $1,814,328.52$ & 6.84 \\
Case 4 & $11,403,986$ & $141,878.60$ & $1,719,403.43$ & 7.30 \\
\hline
\end{tabular}

Table 6: Analysis of the ROI for the Recife feeder installation

\begin{tabular}{l|r|r|r|r}
\hline Scenario & Invest $(\mathrm{R} \$)$ & Maint. $(\mathrm{R} \$ / \mathrm{yr})$ & Annual inc $(\mathrm{R} \$)$ & $\mathrm{ROI}(\mathrm{yrs})$ \\
\hline \hline Case 1 & $11,413,266$ & $142,806.60$ & $1,895,603.25$ & 6.55 \\
Case 2 & $11,408,626$ & $142,342.60$ & $1,852,504.37$ & 6.68 \\
Case 3 & $11,411,979$ & $142,677.90$ & $1,778,140.46$ & 7.06 \\
Case 4 & $11,403,986$ & $141,878.60$ & $1,674,316.05$ & 7.49 \\
\hline
\end{tabular}

It can be seen that the recovered revenue grows as the number of installed DG and CB increases. The expected ROI is around 6-7 years, which is adequate, especially considering the useful life of wind generators and photovoltaic panels, which is around 20 years (without considering the converters).

\section{Conclusions}

This paper first presented a methodology to obtain the probabilistic model of electric energy generation through wind generators and photovoltaic panels. The model consists of the load prediction through the historical series correlation of the wind speed and the solar irradiation of a specific region (Recife, Brazil). In order to preserve the seasonality, the year is divided into 96 hourly intervals, representing the 24 hours of the day of the four seasons. In addition, a technique to obtain the optimal location of the Distributed Generation Units is proposed using a meta-heuristic Genetic Algorithm. The optimization aims to minimize the annual losses of active power and the overall cost of investment, taking into account the inherent constraints of the problem. 
To validate the approach, several case studies were analyzed using data from two feeders from the LIGHT distribution network located in Rio de

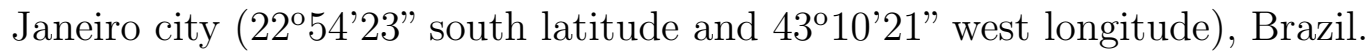
For an analysis closer to reality, the worst-case demand values were used when the network overloaded. The network is composed of 60 wind generators and 6000 photovoltaic panels, equally distributed among the Distributed Generation Units. This makes it possible to analyze the influence of the distribution of these generators in the feeders bars. Capacitor Banks were used, as they contribute to regulating the voltage at suitable levels.

The results showed that the proposed methodology to optimize the allocation of the Distributed Generation Units and Capacitor Banks significantly reduces the losses of active power in the system. Among the different test scenarios, those with the highest number of Distributed Generation Units obtained lower losses and a higher financial return. The expectation of the return on investment was estimated to be between 6 and 7 years, which is quite satisfactory.

In summary, it has been shown that the proposed methodology for load forecasting and optimizing the Distributed Generation Units allocation can be an important tool for planning electric power systems. This methodology deals with the stochastic nature of the Distributed Generation Units through a probabilistic model, with the aim of improving the technical and operational performance of the distribution networks with the proper allocation of Distributed Generation Units.

Finally, it should be stressed that the methodology proposed in this paper is universal, and can be applied to other feeders. Its application requires only

the measurements of a local weather station, and the consumption history of the feeder."

\section{Acknowledgment}

The authors acknowledge the support from the CNPq 309823/2018-8, FAPES-2021-WMR44 projects and NiDA. Prof. Tadeo is funded by the Junta de Castilla y León and EU-FEDER funds (CLU-2017-09, VA232P18, UIC 225). Dr. Imene Yahyaoui is funded by RESOL Project (M2421, URJC).

\section{References}

[1] J. F. Fardin, H. R. de Oliveira Rocha, C. B. Donadel, R. Fiorotti, Distributed generation energy in relation to renewable energy: Principle, 
techniques, and case studies, in: I. Yahyaoui (Ed.), Advances in Renewable Energies and Power Technologies, Elsevier, 2018, pp. 345-375.

[2] T. Ackermann, G. Andersson, L. Söder, Distributed generation: a definition, Electric power systems research 57 (3) (2001) 195-204.

[3] K. B. Debnath, M. Mourshed, Forecasting methods in energy planning models, Renewable and Sustainable Energy Reviews 88 (2018) 297-325.

[4] R. Fiorotti, J. Fardin, L. Encarnacao, C. Donadel, A novel strategy for distribution network reinforcement planning considering the firm capacity of distributed generation units, IEEE Latin America Transactions 17 (04) (2019) 530-539.

[5] M. Zhao, H. Hui, W. Liu, J. Su, Time-varying characteristic based load forecasting method for distribution network with high penetration dgs, in: AORC-CIGRE Technical Meeting 2013, 2013.

[6] G. Papaefthymiou, D. Kurowicka, Using copulas for modeling stochastic dependence in power system uncertainty analysis, IEEE Transactions on Power Systems 24 (1) (2008) 40-49.

[7] A. R. Jordehi, Allocation of distributed generation units in electric power systems: A review, Renewable and Sustainable Energy Reviews 56 (2016) 893-905.

[8] M. A. Nezhadpashaki, F. Karbalaei, S. Abbasi, Optimal placement and sizing of distributed generation with small signal stability constraint, Sustainable Energy, Grids and Networks 23 (2020) 100380.

[9] S.-E. Razavi, E. Rahimi, M. S. Javadi, A. E. Nezhad, M. Lotfi, M. Shafiekhah, J. P. Catalão, Impact of distributed generation on protection and voltage regulation of distribution systems: A review, Renewable and Sustainable Energy Reviews 105 (2019) 157-167.

[10] Z. Abdmouleh, A. Gastli, L. Ben-Brahim, M. Haouari, N. A. Al-Emadi, Review of optimization techniques applied for the integration of distributed generation from renewable energy sources, Renewable Energy 113 (2017) 266-280. 
[11] A. Zakaria, F. B. Ismail, M. H. Lipu, M. A. Hannan, Uncertainty models for stochastic optimization in renewable energy applications, Renewable Energy 145 (2020) 1543-1571.

[12] A. Ehsan, Q. Yang, Optimal integration and planning of renewable distributed generation in the power distribution networks: A review of analytical techniques, Applied Energy 210 (2018) 44-59.

[13] K. H. Truong, P. Nallagownden, I. Elamvazuthi, D. N. Vo, A quasioppositional-chaotic symbiotic organisms search algorithm for optimal allocation of $\mathrm{dg}$ in radial distribution networks, Applied Soft Computing 88 (2020) 106067.

[14] O. Sadeghian, A. Oshnoei, R. Khezri, S. Muyeen, Risk-constrained stochastic optimal allocation of energy storage system in virtual power plants, Journal of Energy Storage 31 (2020) 101732.

[15] M. Barukčić, T. Varga, V. Jerković Štil, T. Benšić, Co-simulation framework for optimal allocation and power management of dgs in power distribution networks based on computational intelligence techniques, Electronics 10 (14) (2021) 1648.

[16] E. A. Al-Ammar, G. A. Ghazi, W. Ko, Y. Khan, A. Beroual, J. Hong, S.-H. Song, Comprehensive impact analysis of ambient temperature on multi-objective capacitor placements in a radial distribution system, Ain Shams Engineering Journal (2020).

[17] S. K. Injeti, V. K. Thunuguntla, M. Shareef, Optimal allocation of capacitor banks in radial distribution systems for minimization of real power loss and maximization of network savings using bio-inspired optimization algorithms, International Journal of Electrical Power \& Energy Systems 69 (2015) 441-455.

[18] A. Rahiminejad, S. H. Hosseinian, B. Vahidi, S. Shahrooyan, Simultaneous distributed generation placement, capacitor placement, and reconfiguration using a modified teaching-learning-based optimization algorithm, Electric Power Components and Systems 44 (14) (2016) 1631-1644.

[19] B. R. Pereira, G. R. M. da Costa, J. Contreras, J. R. S. Mantovani, Optimal distributed generation and reactive power allocation in electrical 
distribution systems, IEEE Transactions on Sustainable Energy 7 (3) (2016) 975-984.

[20] P. Kayal, C. Chanda, Strategic approach for reinforcement of intermittent renewable energy sources and capacitor bank for sustainable electric power distribution system, International Journal of Electrical Power \& Energy Systems 83 (2016) 335-351.

[21] M. Kumar, P. Nallagownden, I. Elamvazuthi, Optimal placement and sizing of renewable distributed generations and capacitor banks into radial distribution systems, Energies 10 (6) (2017) 811.

[22] K. Muthukumar, S. Jayalalitha, Integrated approach of network reconfiguration with distributed generation and shunt capacitors placement for power loss minimization in radial distribution networks, Applied Soft Computing 52 (2017) 1262-1284.

[23] L. Zhang, C. Shen, Y. Chen, S. Huang, W. Tang, Coordinated allocation of distributed generation, capacitor banks and soft open points in active distribution networks considering dispatching results, Applied energy 231 (2018) 1122-1131.

[24] A. A. Abou El-Ela, R. A. El-Sehiemy, A. S. Abbas, Optimal placement and sizing of distributed generation and capacitor banks in distribution systems using water cycle algorithm, IEEE Systems Journal 12 (4) (2018) 3629-3636.

[25] A. Bayat, A. Bagheri, Optimal active and reactive power allocation in distribution networks using a novel heuristic approach, Applied Energy 233 (2019) 71-85.

[26] S. Ouali, A. Cherkaoui, Optimal allocation of combined renewable distributed generation and capacitor units for interconnection cost reduction, Journal of Electrical and Computer Engineering, Article ID 5101387 (2020) 1-11.

[27] N. Kanwar, N. Gupta, K. Niazi, A. Swarnkar, R. Bansal, Simultaneous allocation of distributed energy resource using improved particle swarm optimization, Applied Energy 185 (2017) 1684-1693. 
[28] A. M. Shaheen, R. A. El-Sehiemy, Optimal coordinated allocation of distributed generation units/capacitor banks/voltage regulators by egwa, IEEE Systems Journal 15 (1) (2020) 257-264.

[29] C. Venkatesan, R. Kannadasan, M. H. Alsharif, M.-K. Kim, J. Nebhen, A novel multiobjective hybrid technique for siting and sizing of distributed generation and capacitor banks in radial distribution systems, Sustainability 13 (6) (2021) 3308.

[30] A. Naderipour, Z. Abdul-Malek, M. Hajivand, Z. M. Seifabad, M. A. Farsi, S. A. Nowdeh, I. F. Davoudkhani, Spotted hyena optimizer algorithm for capacitor allocation in radial distribution system with distributed generation and microgrid operation considering different load types, Scientific reports 11 (1) (2021) 1-15.

[31] A. Abdelaziz, Y. Hegazy, W. El-Khattam, M. Othman, Optimal allocation of stochastically dependent renewable energy based distributed generators in unbalanced distribution networks, Electric Power Systems Research 119 (2015) 34-44.

[32] Q. Zhao, P. Wang, L. Goel, Y. Ding, Evaluation of nodal reliability risk in a deregulated power system with photovoltaic power penetration, IET Generation, Transmission \& Distribution 8 (3) (2013) 421-430.

[33] S. Kaur, G. Kumbhar, J. Sharma, A minlp technique for optimal placement of multiple dg units in distribution systems, International Journal of Electrical Power \& Energy Systems 63 (2014) 609-617.

[34] G. Mokryani, P. Siano, Evaluating the integration of wind power into distribution networks by using monte carlo simulation, International Journal of Electrical Power \& Energy Systems 53 (2013) 244-255.

[35] S. H. Jangamshetti, V. G. Rau, Site matching of wind turbine generators: a case study, IEEE Transactions on Energy Conversion 14 (4) (1999) $1537-1543$.

[36] J. Liu, W. Fang, Y. Yang, C. Yang, S. Lei, S. Fu, Increasing wind power penetration level based on hybrid wind and photovoltaic generation, in: 2013 IEEE International Conference of IEEE Region 10 (TENCON 2013), IEEE, 2013, pp. 1-5. 
[37] V. Thapar, G. Agnihotri, V. K. Sethi, Critical analysis of methods for mathematical modelling of wind turbines, Renewable Energy 36 (11) (2011) 3166-3177.

[38] H. R. Rocha, I. H. Honorato, R. Fiorotti, W. C. Celeste, L. J. Silvestre, J. A. Silva, An artificial intelligence based scheduling algorithm for demand-side energy management in smart homes, Applied Energy 282 (2021) 116145.

[39] Y. Atwa, E. El-Saadany, M. Salama, R. Seethapathy, Optimal renewable resources mix for distribution system energy loss minimization, IEEE Transactions on Power Systems 25 (1) (2009) 360-370.

[40] M. R. Qader, I. S. Qamber, Long-term load forecasting for the kingdom of bahrain using monte carlo method, Journal of the Association of Arab Universities for Basic and Applied Sciences 9 (1) (2010) 12-17.

[41] R. Broadwater, A. Chandrasekaran, C. Huddleston, A. Khan, Power flow analysis of unbalanced multiphase radial distribution systems, Electric Power Systems Research 14 (1) (1988) 23-33.

[42] A. A. Augusto, J. C. S. de Souza, M. B. Do Coutto Filho, H. R. de Oliveira Rocha, J. E. V. Tafur, Optimized capacitor placement considering load and network variability, Journal of Control, Automation and Electrical Systems 31 (6) (2020) 1489-1498.

[43] D. E. Goldberg, J. H. Holland, Genetic algorithms and machine learning, Kluwer Academic Publishers (1988). 